\title{
Binary integration nonparametric detection for range-spread targets in distributed terahertz radar network under unknown clutter
}

\author{
Tong Liu ${ }^{1}$, Rui Min ${ }^{1 *}$, Yiming $\mathrm{Pi}^{1}$, Keyu Long ${ }^{2}$ and Zhongtao Huang ${ }^{2}$
}

\begin{abstract}
In this study, to detect person-borne concealed threats in range profiles under the circumstance of unknown clutter, we propose a binary integration nonparametric detection method based on the generalized sign (GS) detector for range-spread targets in a distributed terahertz radar network (DTRN). In the detection, the length of range-spread targets and the number of dominant scatterers on range-spread targets are considered and adaptively estimated. Furthermore, the GS detection method is applied to maintain a constant false alarm rate (CFAR) under the circumstance of unknown clutter. The detection performance of the proposed method for single terahertz radar and DTRN are both examined with the data synthesized by real range-spread targets data and real clutter data. Experimental results show that the proposed method is effective, and for a given false alarm probability, the DTRN exhibits better detection performance than the single terahertz radar.
\end{abstract}

Keywords: Binary integration detection, Nonparametric, Range-spread targets, Distributed terahertz radar network, Unknown clutter

\section{Introduction}

Given its short detection range and penetrativity, terahertz radar is mainly applied in the fields of security checks and anti-terrorism with high-resolution screening imaging at a stand-off distance [1-3]. Meanwhile, terahertz radar can also detect concealed threats in range profiles (the real range profile of a metallic pistol model beneath clothing is obtained by $0.34 \mathrm{THz}$ radar, as shown in Fig. 1). Compared with high-resolution screening imaging, the concealed threat detection in range profiles can observe a large area and requires a short time. However, as the viewing angle of a single radar is limited, a radar network is needed in a large-scale sence. As shown in Fig. 2, a circular terahertz radar network is adopted to observe a $360^{\circ}$ view of the scene further. A certain number of sensors that observe the same scope in the sence constitute a

*Correspondence: minrui@uestc.edu.cn

${ }^{1}$ School of Electronic Engineering, University of Electronic Science and Technology of China, No. 2006 Xi Yuan Avenue, Chengdu, China Full list of author information is available at the end of the article
DTRN for distributed detection. The radar network consists of several DTRNs, which monitor concealed threats in all aspects.

Concealed threats are range-spread targets for highresolution radar. In order to solve the so-called collapsing loss which will lead to a performance loss for range-spread targets detection [4], many detectors are presented. Assuming the a priori scatterer density of range-spread targets, a detector dependent of spatial density is investigated [5]. Given that the scattering characteristics of range-spread targets are significantly different, completing the aforementioned method is difficult. When the scattering geometry of range-spread targets is unknown, the adaptive detectors that are based on the improved one-step or two-step generalized likelihood ratio test are proposed under the circumstance of homogeneous or inhomogeneous clutter [6-12]. However, it is difficult to derive a definite distribution model to represent the practical clutter in concealed threat detection, which may consist of radar echoes of the human body, 


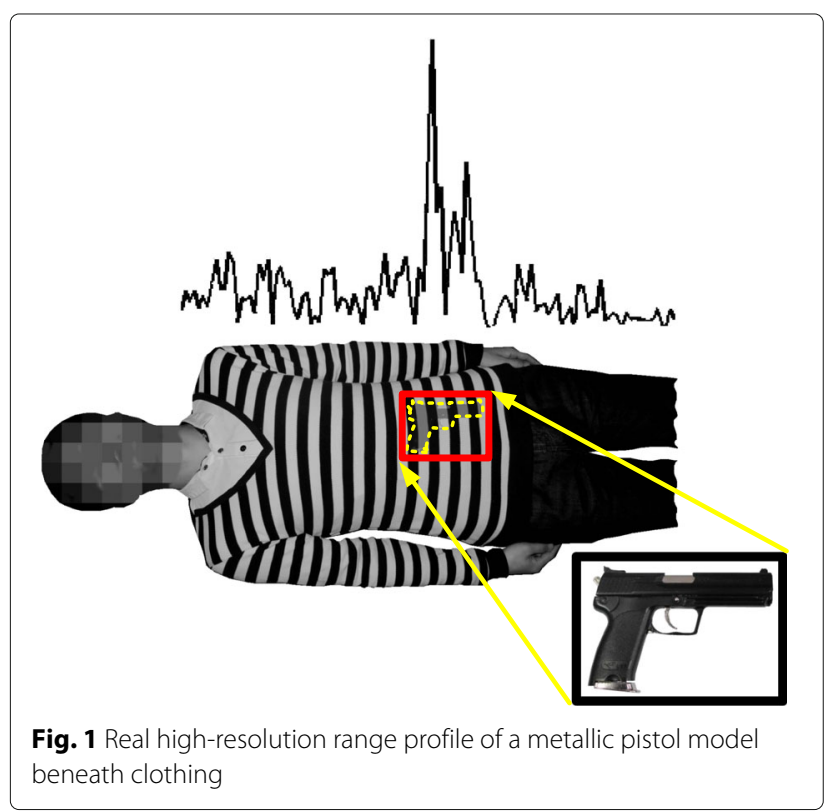

clothing, the ground, and thermal noise. Since those aforementioned detectors are incapable of maintaining the constant false alarm rate (CFAR) to detect concealed threats, nonparametric detectors for range-spread targets are needed. While the traditional nonparametric detectors and their improved counterparts are presented for point target or multiple targets [13, 14]. Research on nonparametric detection for range-spread targets under the circumstance of unknown clutter has rarely been reported. Therefore, a nonparametric detector that considers the knowledge about dominant scatterers on rangespread targets is investigated in this study.

The binary integration nonparametric detector based on the GS detector (BINGS) for range-spread targets in

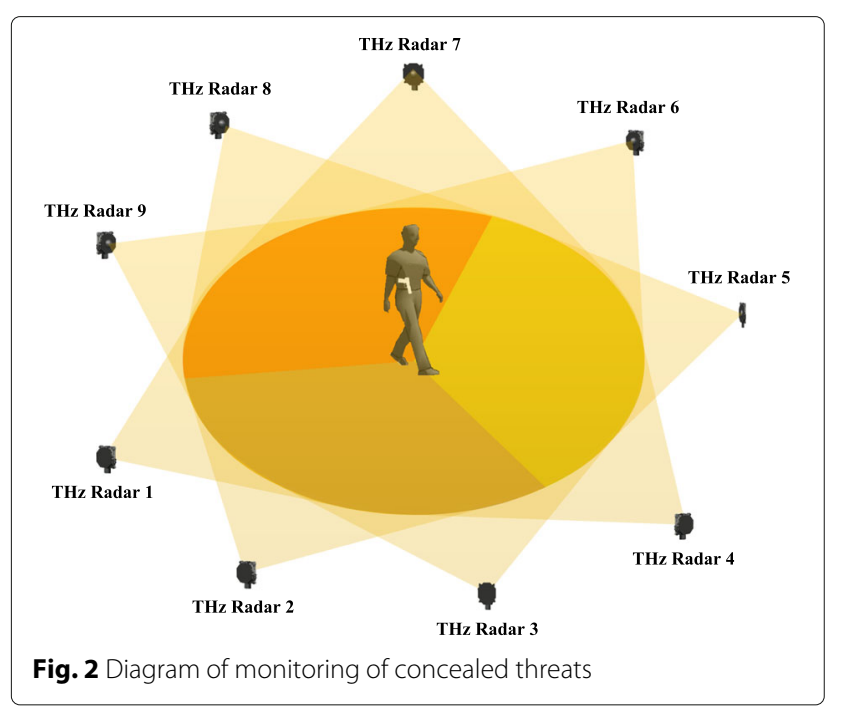

DTRN is presented to satisfy the aforementioned practical application requirements. Given that the length of range cell samples is larger than that of range-spread targets, the sliding window detection method is applied to the local detectors. First of all, the length of the sliding window which is the possible length of range-spread targets is estimated via the maximal signal to clutter ratio (SCR) rule [15]. What is more, the number of dominant scatterers within the sliding window is selected as the threshold for binary integration detection, which is estimated via Otsu's method [16]. Then, after the GS detection of each scatterer within the sliding window, binary integration detection is performed. Finally, the overall decision of DTRN is gained following the $m$-out-of- $n$ fusion rule. The detection performance of single terahertz radar and DTRN is analyzed under the circumstance of unknown clutter by using the data synthesized by real range-spread targets data and real clutter data. Experimental results show that the proposed method is effective, and for a given false alarm probability, the DTRN exhibits better detection performance than the single terahertz radar.

This paper is organized as follows. In Section 2, the structure of the BINGS detector in DTRN is described. In Section 3, the mathematical processes related to detection are derived. In Section 4, the experiments are described and the results are analyzed. In Section 5, the comments and conclusions are presented.

\section{Detector structure}

The BINGS structure is shown in Fig. 3. Terahertz radar transmits the linear frequency modulation signal with large bandwidth. After dechirping of radar echoes, range cell samples are obtained. Because the length of range cell samples is larger than that of range-spread targets, the sliding window detection method is used for range cell samples.

First of all, the length of the sliding window which is the possible length of range-spread targets is estimated via the maximal SCR rule. Then, utilizing $N$ reference cells surround the sliding window, the test statistic of the GS detection $T_{\mathrm{GS}}$ is constructed for each scatterer within the sliding window. At the same time, the first threshold $T_{\mathrm{GSP}}$ for $T_{\mathrm{GS}}$ corresponding to the false alarm probability $P_{f_{\mathrm{GS}}}$ is calculated by Eq. (11). As a result, the sequence of "0" and " 1 " decisions are output by comparing $T_{\mathrm{GS}}$ with $T_{\mathrm{GSP}}$ for each scatterer. So far, the GS detection for each scatterer within the sliding window is completed. In addition, the second threshold QOPT is adaptively estimated by Otsu's method after the sliding window is determined, where $Q_{\mathrm{OPT}}$ is the number of dominant scatterers within the sliding window. Finally, the local binary decision of single detector is obtained by comparing the sum of the sequence of " 0 " and " 1 " with QOPT. 


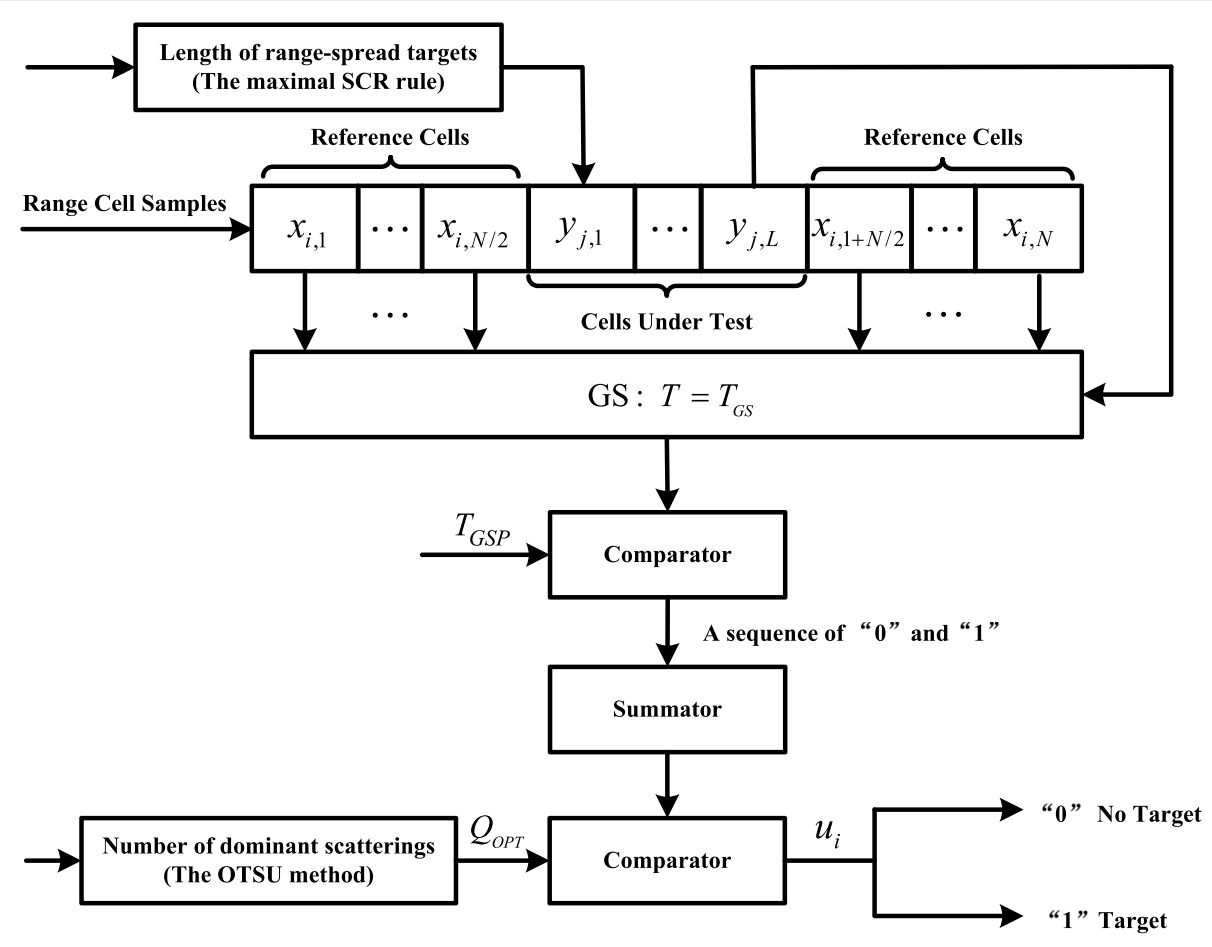

Fig. 3 Flow chart of the BINGS

The block diagram of the DTRN data fusion is shown in Fig. 4. DTRN is formed from $n$ local BINGS detectors which are considered independent and a data fusion center. The local binary decisions are transmitted from the local detectors to the data fusion center. The overall decision is obtained following the $m$-out-of- $n$ fusion rule.

\section{Theory and methodology}

\subsection{Length of range-spread targets estimation}

Regardless of whether the range-spread targets or the attitude angles of the same range-spread target are different, the length of range-spread targets varies significantly, which has an important effect on the detection performance. Here, the length of range-spread targets will be adaptively estimated via the maximal SCR rule [15].

The sliding window detection method is used for the range-spread targets detection. The length of range cell samples denoted by $x(i), i=1,2, \cdots, Z$ is $Z$. The sliding window is $w=[k, k+L-1]$, where $k$ and $L$ are the starting range cell and the length of the sliding window, respectively. Assuming that the entire range-spread target is inside the sliding window and all the range cells outside

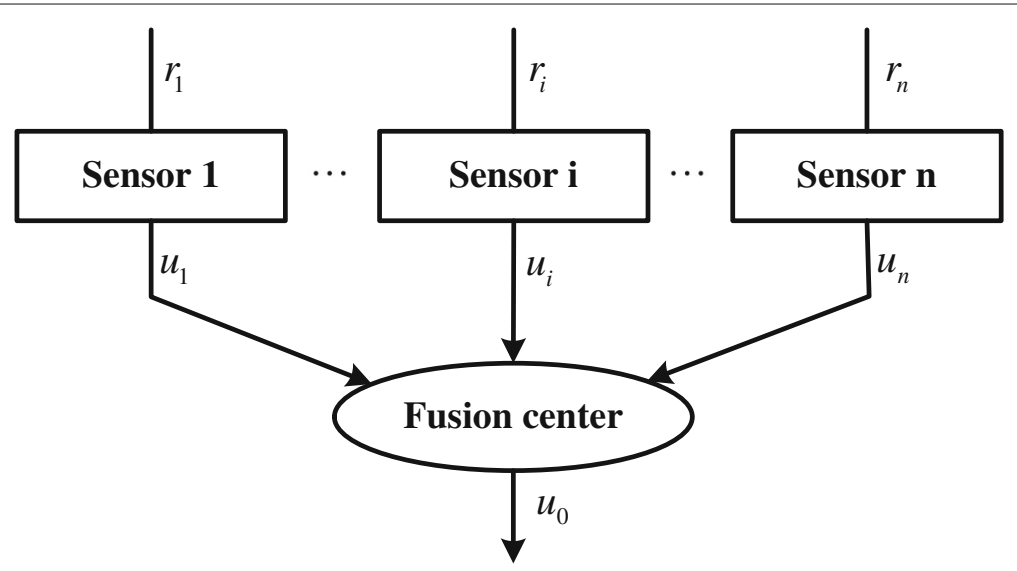

Fig. 4 Block diagram of DTRN data fusion 
of the sliding window are clutter, the average SCR can be defined as follows:

$$
\operatorname{ASCR}(k, L)=\frac{\frac{1}{L} \sum_{i=k}^{k+\mathrm{L}-1}|x(i)|^{2}}{\frac{1}{Z-L}\left(\sum_{i=1}^{k-1}|x(i)|^{2}+\sum_{i=k+L}^{\mathrm{Z}}|x(i)|^{2}\right)} .
$$

The starting range cell $k$ and the length $L$ of rangespread targets are unknown. While the possible minimal length $L_{\min }$ and maximal length $L_{\max }$ of range-spread targets are known.

The window slides over the range profiles. For each starting range cell $k$, the width of the sliding window $\Delta w$ is adjusted between $L_{\min }$ and $L_{\max }$. The length of the sliding window can be estimated by the maximal SCR rule

$$
\bar{L}=\arg \left(\max _{L_{\min } \leq L \leq L_{\max }, 1 \leq k \leq Z-L}(\operatorname{ASCR}(k, L))\right)
$$

where $\bar{L}$ is the estimation value of the possible number of range cell samples that range-spread targets occupy.

\subsection{Number of dominant scatterer estimation}

The radar echo energy of range-spread targets is mainly distributed in dominant scatterers. Hence, the distribution of dominant scatterers on range-spread targets has an important effect on the detection performance. The number of dominant scatterers within the sliding window is used as the threshold for binary integration detection.

Similar to Otsu's method initially utilized in picture processing [16], the number of dominant scatterers within the sliding window is adaptively estimated. When the variance between various classifications is maximal, the range cells within the sliding window are divided into dominant scatterers and the others (small scatterers that can be regarded as clutter and/or clutter) according to amplitude. And in this situation, the probability of false segmentation is minimal.

The amplitude of $L$ range cells inside the sliding window are normalized and sorted in descending order. The new sequence $x^{\prime}(i)$ is obtained as follows:

$$
x^{\prime}(1) \geq x^{\prime}(2) \geq \cdots \geq x^{\prime}(L)>0,
$$

where $x^{\prime}(i)$ is divided into two classes, namely, $C_{1}$ (dominant scatterers) and $C_{2}$ (small scatterers that can be regard as clutter and/or clutter) by a threshold $T_{t b}$. The average amplitude of $C_{1}$ and $C_{2}$ are given by

$$
\left\{\begin{array}{l}
u_{1}=\frac{1}{L_{1}} \sum_{i \in C_{1}} x^{\prime}(i) \\
u_{2}=\frac{1}{L_{2}} \sum_{i \in C_{2}} x^{\prime}(i)
\end{array}\right.
$$

where $L_{1}$ and $L_{2}$ are the sample number of $C_{1}$ and $C_{2}$, respectively. The average amplitude of $x^{\prime}(i)$ is denoted by $u$, and the variance between $C_{1}$ and $C_{2}$ is denoted by $\sigma_{b}^{2}$.

$$
\begin{gathered}
u=\omega_{1} * u_{1}+\omega_{2} * u_{2} \\
\sigma_{b}^{2}=\omega_{1} *\left(u_{1}-u\right)^{2}+\omega_{2} *\left(u_{2}-u\right)^{2},
\end{gathered}
$$

where $\omega_{1}=L_{1} / L$ and $\omega_{2}=L_{2} / L$. Plugging Eqs. (4) and (5) into Eq. (6) yields

$$
\sigma_{b}^{2}=\frac{L_{1} * L_{2}}{L^{2}}\left(\frac{1}{L_{1}} \sum_{i \in C_{1}} x^{\prime}(i)-\frac{1}{L_{2}} \sum_{i \in C_{2}} x^{\prime}(i)\right)^{2} .
$$

The optimal threshold $T_{b}$ that maximizes $\sigma_{b}^{2}$ can be selected as follows:

$$
T_{b}=\arg \left(\max _{T_{t b} \in\left\{x^{\prime}(i), i=1,2, \cdots, L-1\right\}}\left\{\sigma_{b}^{2}\right\}\right) .
$$

$T_{b}$ is obtained by searching the one-dimensional parameter space. Then, the number of scatterers whose amplitute is greater than or equal to $T_{b}$ within the sliding window is estimated as the optimal threshold $Q_{O P T}$.

\subsection{Test statistic of single detector}

$y_{j, p}(j=1,2, \cdots, M, p=1,2, \cdots, L)$ denotes the range cell samples under the test, where $M$ is the number of integration pulses. $x_{i, q}(i=1,2, \cdots M, q=1,2, \cdots, N)$ denotes the reference cell samples, where $N$ is the number of reference cells. The test statistic of the GS detection for each range cell within the sliding window is given by

$$
T_{G S}=\sum_{j=1}^{M} r_{j}=\sum_{j=1}^{M} \sum_{i=1}^{N} u\left(y_{j p}-x_{j i}\right),
$$

where $r_{j}=\sum_{i=1}^{N} u\left(y_{j p}-x_{j i}\right)$ is the rank of the single scatterer under test, $u\left(y_{j p}-x_{j i}\right)=\left\{\begin{array}{ll}1, & y_{j p} \geq x_{j i} \\ 0, & y_{j p}<x_{j i}\end{array}\right.$.

The corresponding detection strategy is

$$
\left\{\begin{array}{ll}
T_{\mathrm{GS}} \geq T_{\mathrm{GSP}}, & \text { scatterer } \\
T_{\mathrm{GS}}<T_{\mathrm{GSP}}, & \text { no scatterer }
\end{array},\right.
$$

where $T_{\mathrm{GSP}}$ is the threshold for the single scatterer GS detection that corresponds to the false alarm probability $P_{f_{G S}}$, which is derived as follows [17]:

$$
\begin{aligned}
P_{f_{\mathrm{GS}}} & =P_{r}\left(T_{\mathrm{GS}} \geq T_{\mathrm{GSP}} \mid H_{0}\right) \\
& =\sum_{k=T_{\mathrm{GSP}}}^{M N} P_{r}\left(T_{\mathrm{GS}}=k \mid H_{0}\right),
\end{aligned}
$$

where the scatterer is absent under hypothesis $H_{0}$ and the scatterer is present under hypothesis $H_{1}$.

The probability density distribution function of clutter is $c(t)$ and that of clutter plus the signal is $s_{-} c(t)$. Assuming that the clutter samples are independent and 
identically distributed (i.i.d.), the probability density distribution of $r_{j}=r$ can be expressed as

$$
P_{r}\left(r_{j}=r\right)=C_{N}^{r} \int \mathrm{c}^{r}(t)[1-\mathrm{c}(t)]^{N-r} d s_{c}(t),
$$

where $r=0,1, \cdots, N$.

When the range cell under test is the pure clutter, Eq. (12) can be simplified as

$$
P_{r}\left(r_{j}=r\right)=\frac{1}{N+1}, 0 \leq r \leq N .
$$

The generating function of $r_{j}$ is

$$
G S_{j}(t)=\sum_{r=0}^{N}\left(\frac{1}{N+1}\right) t^{r} .
$$

$r_{j}(j=1,2, \cdots, M)$ is an i.i.d. random variable. The generating function of $T_{\mathrm{GS}}$ is given by

$$
G S(t)=\left[G S_{j}(t)\right]^{M}
$$

Then,

$$
P_{r}\left[T_{G S}=k \mid H_{0}\right]=\left.\frac{1}{k !} \frac{d^{k} G S(t)}{d t^{k}}\right|_{t=0}
$$

Equation (16) can be rewritten as

$$
P_{r}\left[T_{\mathrm{GS}}=k \mid H_{0}\right]=\left.\frac{1}{(N+1)^{M}} \bullet \frac{d^{k}\left(\sum_{r=0}^{N} t^{r}\right)^{M}}{k ! d t^{k}}\right|_{t=0} .
$$

The second term in Eq. (17) is equivalent to the coefficient of $t^{k}$.

Equation (17) can be interpreted by combination and mathematical statistics. Assuming that $M$ sets are present and every set is $\{0,1, \cdots, N\}$, one integer is taken from each set and $M$ integers are obtained. $(N+1)^{M}$ is equivalent to the number of all the possible combinations. The second term is equivalent to the number of combinations in which the sum of these $M$ integers is $k$. Therefore, $P_{r}\left[T_{\mathrm{GS}}=k \mid H_{0}\right]$ is the probability of $T_{\mathrm{GS}}=k$ under hypothesis $H_{0}$.

If the values of $M$ and $N$ are set, then the relationship between $P_{f_{\mathrm{GS}}}$ and $T_{\mathrm{GSP}}$ can be determined. Table 1 shows the relationship between $P_{f_{\mathrm{GS}}}$ and $T_{\mathrm{GSP}}$ under the condition of $M=4$ and $N=12$.

For a given false alarm probability $P_{f_{\mathrm{GS}}}, T_{\mathrm{GSP}}$ can be calculated. Then, the detection probability of the single scatterer GS detection can be given by

$$
\begin{aligned}
P_{d_{\mathrm{GS}}} & =P_{r}\left(T_{\mathrm{GS}} \geq T_{\mathrm{GSP}} \mid H_{1}\right) \\
& =\sum_{k=T_{\mathrm{GSP}}}^{M N} \mathrm{P}_{r}\left(T_{\mathrm{GS}}=k \mid H_{1}\right)
\end{aligned}
$$

Table 1 Relationship between $P_{f_{G S}}$ and $T_{G S P}$ under the condition of $M=4$ and $N=12$

\begin{tabular}{lllll}
\hline$P_{f_{G S}}$ & 0.000035 & 0.000175 & 0.000525 & 0.0012 \\
$T_{G S P}$ & 48 & 47 & 46 & 45 \\
$P_{f_{G S}}$ & 0.0025 & 0.0044 & 0.0074 & 0.0116 \\
$T_{G S P}$ & 44 & 43 & 42 & 41 \\
$P_{f_{G S}}$ & 0.0173 & 0.0250 & 0.0350 & 0.0478 \\
$T_{G S P}$ & 40 & 39 & 38 & 37 \\
$P_{f_{G S}}$ & 0.0637 & 0.0832 & 0.1064 & 0.1437 \\
$T_{G S P}$ & 36 & 35 & 34 & 33 \\
\hline
\end{tabular}

The test statistic of the BINGS detetion is expressed as follows:

$$
\begin{aligned}
T_{\mathrm{BGS}} & =\sum_{p=1}^{L} \operatorname{sgn}\left(T_{\mathrm{GS}}-T_{\mathrm{GSP}}\right) \\
& =\sum_{p=1}^{L} \operatorname{sgn}\left(\sum_{j=1}^{M} \sum_{i=1}^{2 N} u\left(y_{j p}-x_{j i}\right)-T_{\mathrm{GSP}}\right),
\end{aligned}
$$

where $\operatorname{sgn}(\cdot)$ denotes the sign function.

The false alarm probability $P_{f_{\mathrm{BGS}}}$ of the BINGS detection is derived as follows:

$$
P_{f_{\mathrm{BGS}}}=\sum_{k=Q_{\mathrm{opt}}}^{L} C_{L}^{k} P_{f_{\mathrm{GS}}}^{k}\left(1-P_{f_{\mathrm{GS}}}\right)^{L-k} .
$$

$P_{f_{\mathrm{BGS}}}$ is irrelevant to the distribution of clutter. Meanwhile, the threshold for the test statistic of the BINGS detection is independent of the distribution of clutter. Therefore, this detector is a nonparametric detector.

The detection probability $P_{d_{\mathrm{BGS}}}$ of the BINGS detection is derived as follows:

$$
P_{d_{\mathrm{BGS}}}=\sum_{k=Q_{\mathrm{opt}}}^{L} C_{L}^{k} P_{d_{\mathrm{GS}}}^{k}\left(1-P_{d_{\mathrm{GS}}}\right)^{L-k}
$$

\subsection{Distributed terahertz radar network}

The parallel structure is adopted in DTRN, which is formed from $n$ local detectors (the BINGS detector) and a data fusion center. The local binary decisions are transmitted from the local detectors to the data fusion center. The overall decision is obtained on the basis of the $m$-outof- $n$ fusion rule [17]. Assuming that the local detectors have the same performance characteristic, the false alarm probability of each detector is $P_{f_{\mathrm{BGS}}}$ and the detection probability of each detector is $P_{d_{B G S}}$. When the $m$-out-of$n$ fusion rule is used, the overall false alarm probability of DTRN is given by

$$
P_{F}=\sum_{k=m}^{n} C_{n}^{k} P_{f_{\mathrm{BGS}}}^{k}\left(1-P_{f_{\mathrm{BGS}}}\right)^{n-k}
$$


and the overall detection probability of DTRN is given by

$$
P_{D}=\sum_{k=m}^{n} C_{n}^{k} P_{d_{\mathrm{BGS}}}^{k}\left(1-P_{d_{\mathrm{BGS}}}\right)^{n-k}
$$

If the value of $m$ is 1 , then the $m$-out-of- $n$ fusion rule is the OR fusion rule. If the value of $m$ is $n$, then the $m$-outof- $n$ fusion rule is the AND fusion rule.

\section{Experiments and results}

The terahertz radar system based on a solid-state source is shown in Fig. 5. The system operates at $0.34 \mathrm{THz}$ with a bandwidth of $20 \mathrm{GHz}$ which is adjustable [18].

The range-spread target is a metallic pistol model whose length is $14 \mathrm{~cm}$, as shown in Fig. 1. The metallic pistol model is placed on the foam platform at a distance of $5 \mathrm{~m}$ off the radar. The high-resolution range profiles of the metallic pistol model in different radar views are markedly different, as shown in Fig. 6 . The range profiles of the target are extracted and occupy 33,28 , and 36 range cells in radar views 1,2 , and 3 respectively.

The range profiles of a person in $360^{\circ}$ are considered the clutter data (the ground clutter and thermal noise are included). The range profiles of the 10,000th, 20,000th, 30,000 th, and 40,000th pulse in the clutter data are shown in Fig. 7. Every range profile includes 200 range cells. The clutter is related to the distribution of scatterers on a person.

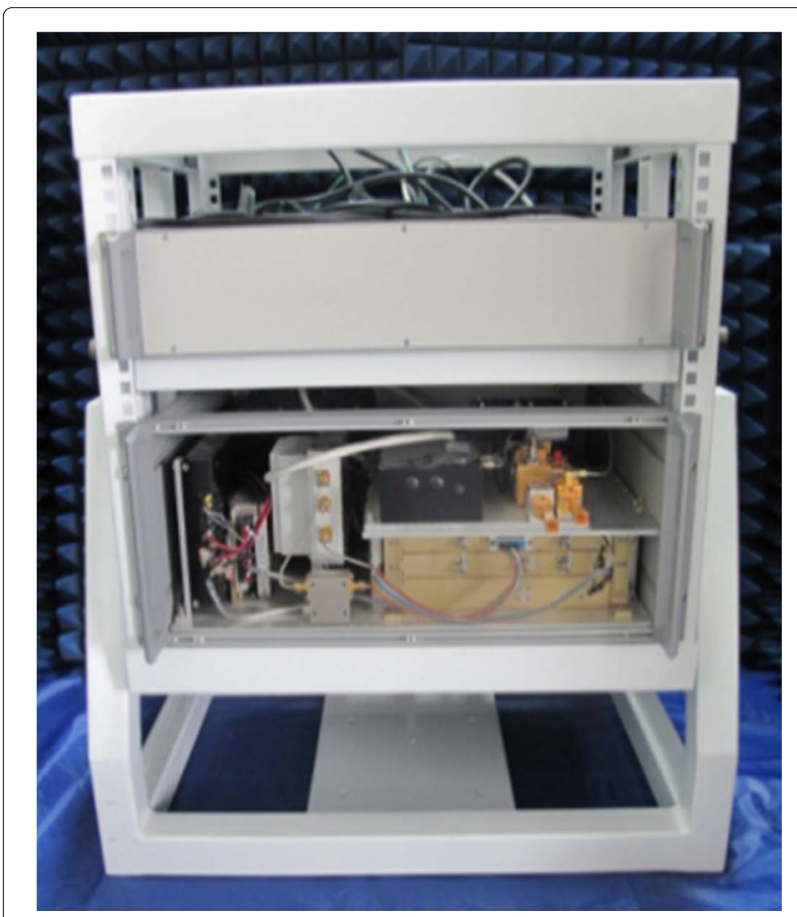

Fig. 5 Image of the $0.34-\mathrm{THz}$ radar system
Rayleigh distribution, gamma distribution, $G_{0}$ distribution, $K$ distribution, Weibull distribution, logarithmic normal distribution, and Nakagami distribution curves are fitted against real clutter data, as shown in Fig. 8. The distribution of real clutter cannot be effectively represented by any of these distribution models. By comparison, the distribution of real clutter data fits the $G_{0}$ distribution model better.

The radar echoes are synthesized by real range-spread targets data and real clutter data. $M$ range profiles are randomly selected from the same group of real range-spread targets data. The range profiles of the range-spread target are extracted from these $M$ range profiles, and each of them is normalized. Meanwhile, $M$ sequential range profiles of clutter are randomly selected from real clutter data and each of them is normalized. $M$ range profiles of the range-spread target and $M$ range profiles of clutter are synthesized into $M$ radar echo pulses. The SCR of each synthesized radar echo pulse is calculated by the following formula:

$$
\mathrm{SCR}=10 \lg \left(\frac{\frac{1}{\mathrm{~L}} \sum_{p=1}^{L}\left|C_{0} y_{j p}\right|^{2}}{\frac{1}{\mathrm{Z}-\mathrm{L}}\left(\sum_{i=1}^{K_{0}-1}\left|x_{j i}\right|^{2}+\sum_{i=K_{0}+L}^{Z}\left|x_{j i}\right|^{2}\right)}\right) \text {, }
$$

where $K_{0}$ is the starting position in which the rangespread target is embedded in the clutter pulse, $j=$ $1,2, \cdots, M$ denotes the $j$ th pulse. The synthesized data in different single-pulse SCRs are obtained by adjusting the value of $C_{0}$, where $C_{0}$ is the overall scaling factor for the amplitude of range-spread targets. $C_{0}$ does not influence the distribution of scatterers on range-spread targets. As shown in Fig. 6, the lengths of the range-spread target in different radar views are estimated by the maximal SCR rule and the estimation values are 31,16 , and 33 , respectively. The number of corresponding dominant scatterers is 7,5 , and 9 respectively. It means that scatterers whose normalized amplitude is more than 0.5 are distinguished as dominant scatterers. Otsu's method is proved to be a valid approach to estimate the number of dominant scatterers on range-spread targets.

When the number of integration pulses and reference cells are different, the detection probability versus the single-pulse SCR for the BINGS detection and the binary integration parametric detector based on cell-averaging (CA)-CFAR (BIPCA-CFAR) detection for the false alarm probability $P_{f_{\mathrm{BGS}}}=10^{-8}$ are shown in Fig. $9 . \mathrm{M}$ is the number of integration pulses. $N$ is the number of reference cells. The pulse repetition frequency is $1000 \mathrm{~Hz}$, and the pulse repetition interval is $1 \mathrm{~ms}$. Considering the slow movement of people, the range between the radar and range-spread targets barely varies in a two-pulse or 


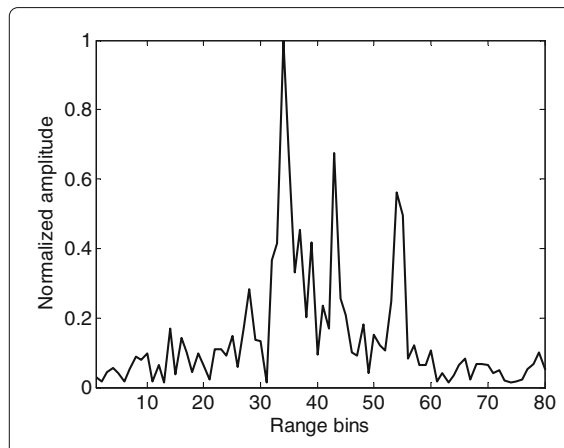

(a)

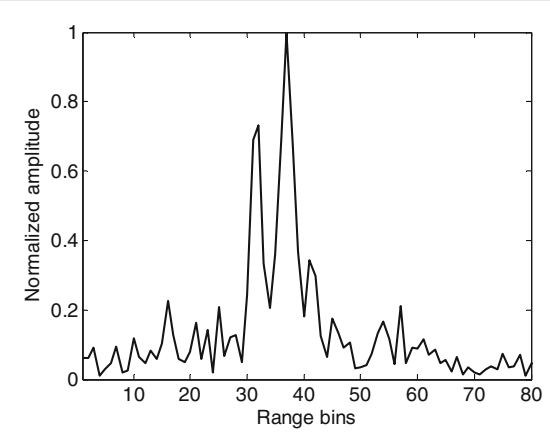

(b)

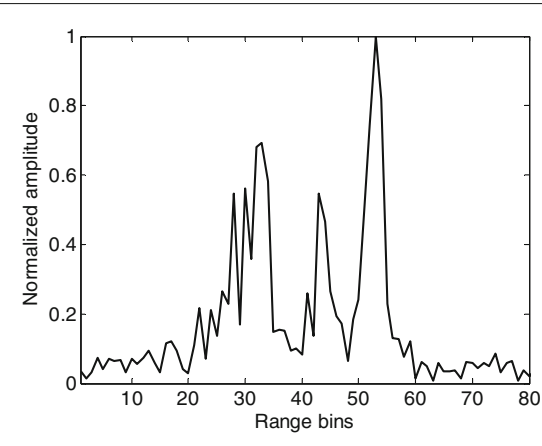

(c)

Fig. 6 Range profiles of the metallic pistol model in different radar views. a Range profile in radar view 1. b Range profile in radar view 2. c Range profile in radar view 3

four-pulse repetition interval. Two or four pulses are considered for integration, which would not lead to range cell migration of range-spread targets. Therefore, setting $M$ as 2 or 4 is reasonable. The BIPCA-CFAR detection is similar to the BINGS detection. The differences are that the CA-CFAR detection is used for each range cell within the sliding window and the distribution of real clutter data is assumed to be the $G_{0}$ distribution model in the BIPCA-CFAR detection.

The BIPCA-CFAR detection is incapable of maintaining CFAR when the assumed distribution model of clutter is inconsistent with the distribution of real clutter, as shown in Fig. 9a. The detection performance of the BINGS detector is better, as shown in Fig. 9b. Comparing the detection performance curves in the case of $M=4, N=$ 12 with the case of $M=2, N=12$ for the BINGS detector, more integration pulses result in the improvement in the detection performance. However, a high number of pulse integrations is not recommended, which will lead to range cell migration. Comparing the detection performance curves in the case of $M=2, N=24$ with the case of $M=2, N=12$ for the BINGS detection, a high number of reference cells leads to degradation of the detection performance. Thus, the number of integration pulses and reference cells should be carefully chosen on the basis of the practical application environment. As

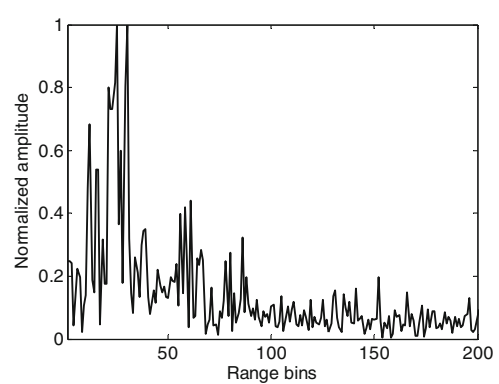

(a)

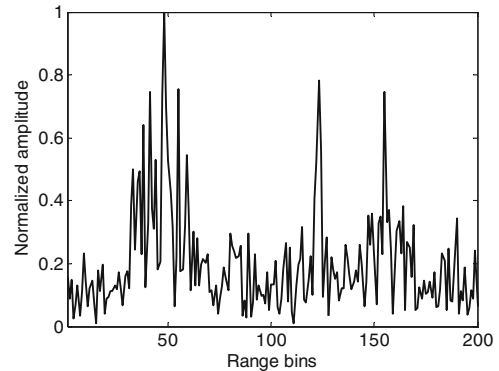

(c)

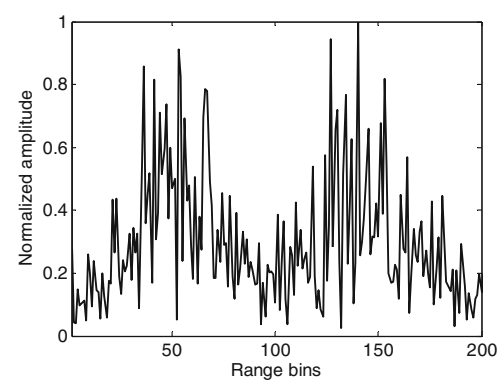

(b)

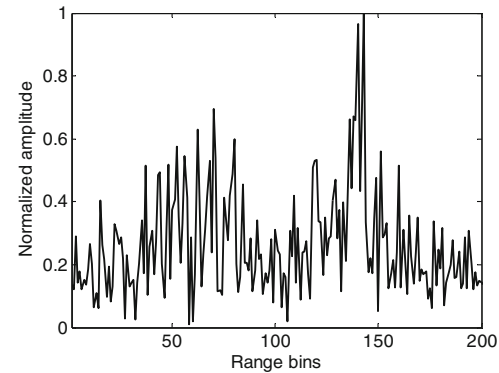

(d)

Fig. 7 Range profiles of a person in different degrees. a Range profile of the 10,000th pulse. b Range profile of the 20,000th pulse. c Range profile of the 30,000 th pulse. $\mathbf{d}$ Range profile of the 40,000 th pulse 


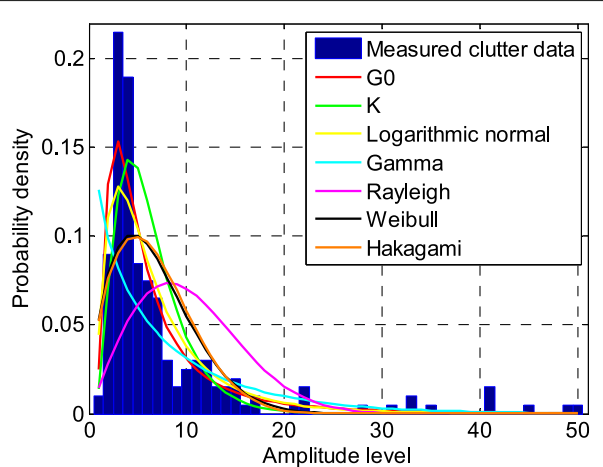

(a)

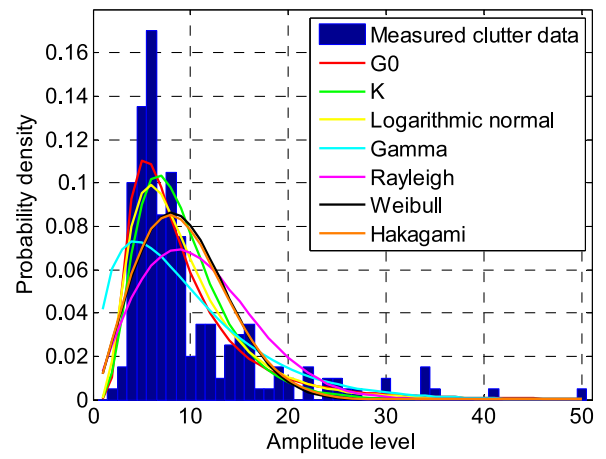

(c)

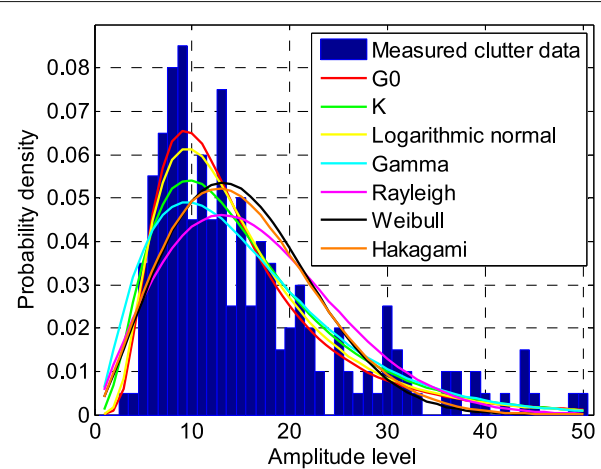

(b)

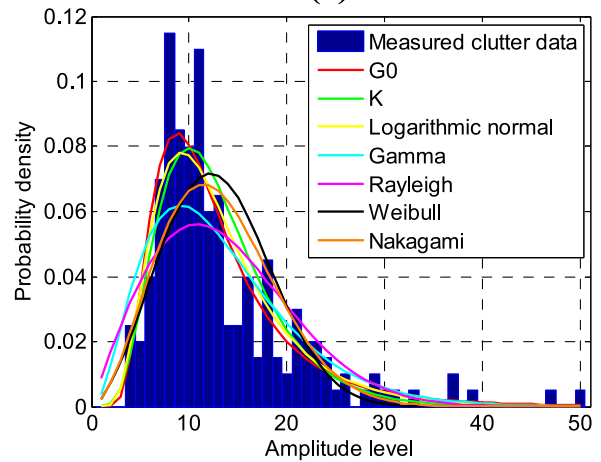

(d)

Fig. 8 Clutter data fit to different distribution models. Amplitude distribution of clutter after the noncoherent integration of a the 10,000th, 10,001 th, 10,002th, and 10,003th pulse; b the 20,000th, 20,001th, 20,002th, and 20,003th pulse; c the 30,000th, 30,001 th, 30,002th, and 30,003th pulse; and $\mathbf{d}$ the 40,000th, 40,001th, 40,002th, and 40,003th pulse

shown in Fig. 9b, the detection performance of the BINGS detector in the case of $M=4, N=12$ is superior.

We assume that DTRN consists of 3 terahertz radars and that the DTRN observes the same range-spread target from different radar views. The BINGS detector is used as the local detector. The single-pulse SCR of these local detectors are equal. The other parameters are set as $P_{F}=10^{-15}, M=4$, and $N=12$. Figure 10 represents the detection performance curves of single radar and DTRN for different fusion rules. The detection performance of DTRN for the AND fusion rule and single radar is poorer than that of DTRN for the 2-out-of-3 fusion rule and the OR fusion rule. The detection probability of DTRN for the AND fusion rule remains at approximately 0.93 when the single-pulse SCR is high. The result shows that the practical false alarm probability of DTRN for the AND fusion

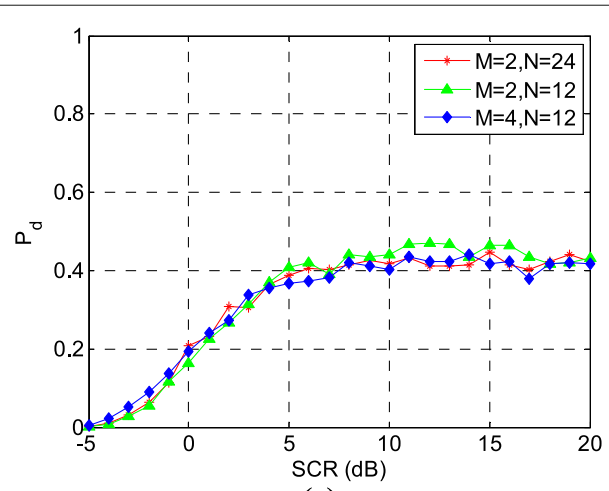

(a)

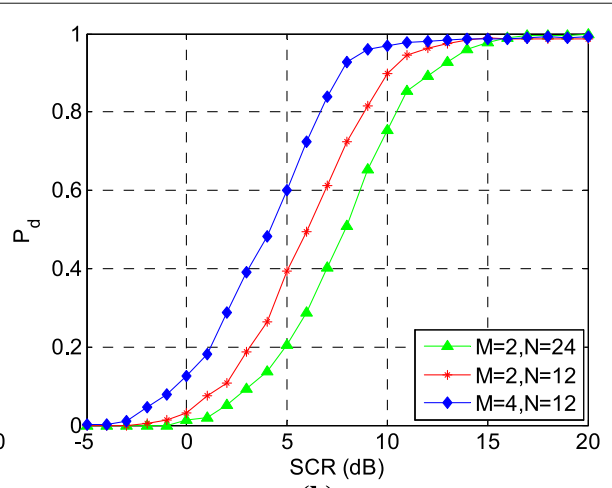

(b)

Fig. 9 Detection performance of two detectors with different numbers of integration pulses and reference cells. a Detection performance curves of the BIPCA-CFAR detector. b Detection performance curves of the BINGS detector 


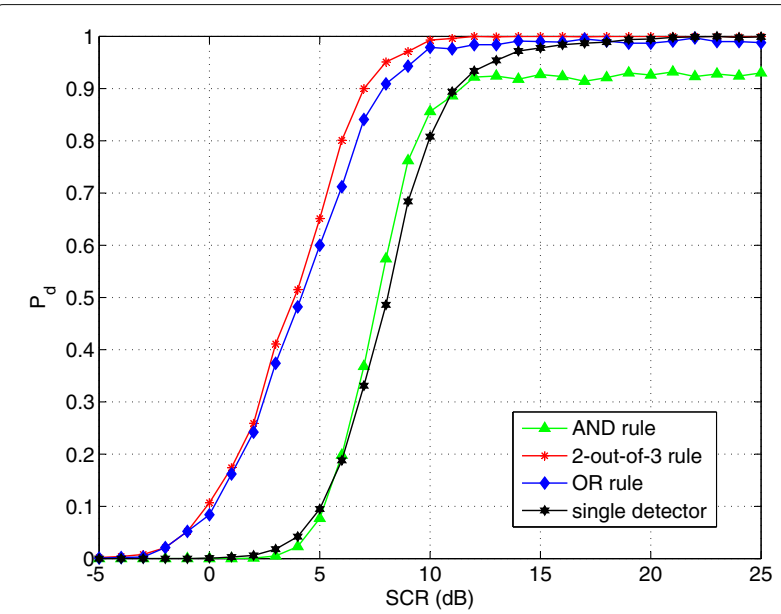

Fig. 10 Detection performance of single radar and DTRN for different fusion rules

rule is higher than the theoretical false alarm probability (the false alarm probability is irrelevant to the single-pulse SCR). The theoretical false alarm probability must be set lower to control the practical false alarm probability.

Given the different detection ranges and radar views, the single-pulse SCR of the local detectors are consistently different. We assume that DTRN consists of 3 terahertz radars and that the DTRN observes the same range-spread target from different radar views. The BINGS detector is used as the local detector. The single-pulse SCR of the two detectors are equal, and the single-pulse SCR of the third detector is less than that of those two detectors by $5 \mathrm{~dB}$. The other parameters are set as $P_{F}=10^{-15}$, $M=4$, and $N=12$. Figure 11 shows the detection performance curves of DTRN for different fusion rules when the SCR of one local detector is different. The horizontal axis represents the single-pulse SCR of two detectors

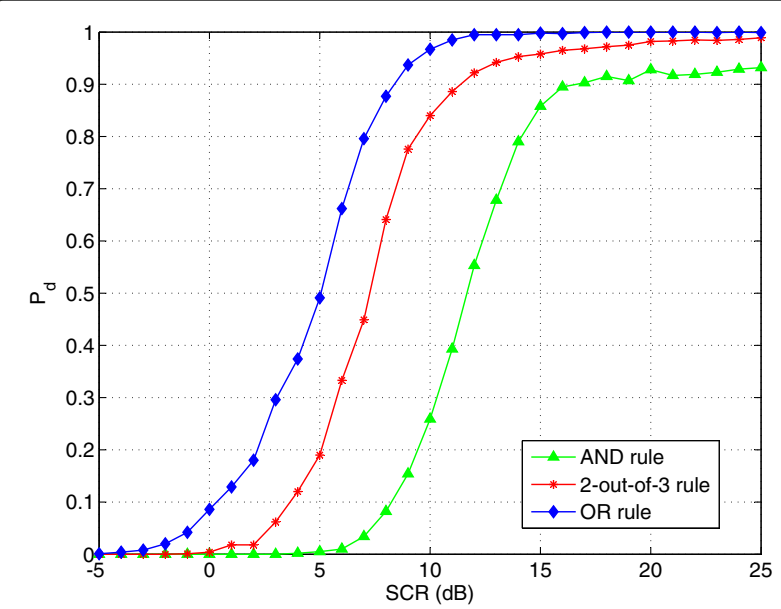

Fig. 11 Detection performance of DTRN when the SCR of one detector is different that have equivalent single-pulse SCR. By comparing the detection performance curves in Fig. 11 with those in Fig. 10, the detection performance loss of DTRN for the AND fusion rule is determined to be more than $4.5 \mathrm{~dB}$. In addition, the detection performance loss of DTRN for the 2-out-of- 3 fusion rule is approximately $3 \mathrm{~dB}$, and the detection performance loss of DTRN for the OR fusion rule is less than $1 \mathrm{~dB}$. The overall decision for the AND fusion rule relies on all the local binary decisions. When the SCR of one of three detectors worsens, the detection performance of DTRN for the AND fusion rule significantly deteriorates. When the SCR of the local detectors are highly different, the OR fusion rule is the optimal fusion rule.

\section{Conclusions}

In this study, a binary integration nonparametric detection method based on the GS detector for range-spread targets in DTRN is proposed to detect person-borne concealed threats in range profiles. Experimental results show that the BINGS detector in DTRN is capable of not only observing range-spread targets from multiple views but also achieving effective detection for concealed threats under the circumstance of unknown clutter. If the single-pulse SCR of the local detectors are almost equal, then the $m$-out-of- $n$ fusion rule is more appropriate for use. Meanwhile, when the single-pulse SCR of the local detectors are markedly different, using the OR fusion rule is recommended. The reasonable choice of the false alarm probability as well as the number of integration pulses and reference cells based on the practical application environment is a particular area of interest.

\section{Acknowledgements}

This work was supported by the National Natural Science Foundation of China under Grants U1433113, the National Nature Science Foundation of China under Grants 61301265, and the Fundamental Research Funds for the Central Universities under Grants ZYGX2015J020.

\section{Competing interests}

The authors declare that they have no competing interests.

\section{Author details}

${ }^{1}$ School of Electronic Engineering, University of Electronic Science and Technology of China, No. 2006 Xi Yuan Avenue, Chengdu, China. ${ }^{2}$ The Second Research Institute of Civil Aviation Administration of China (CAAC), No. 17, Second Section, Second Ring Road South, Chengdu, China.

Received: 30 April 2016 Accepted: 27 October 2016

Published online: 11 November 2016

\section{References}

1. KB Cooper, RJ Dengler, N Llombart, B Thomas, B Thomas, PH Siegel, THz imaging radar for standoff personnel screening. IEEE Trans. Terahertz Sci. Technol. 1(1), 169-182 (2011)

2. S Gu, C Li, X Gao, Z Sun, G Fang, Terahertz aperture synthesized imaging with fan-beam scanning for personnel screening. IEEE Trans. Microw. Theory Tech. 60(12), 3877-3885 (2012)

3. J Grajal, A Badolato, G Rubiocidre, L Ubedamedina, B Menciaoliva, A Garciapino, B Gonzalezvaldes, O Rubinos, 3-D High-resolution imaging 
radar at $300 \mathrm{GHz}$ with enhanced FoV. IEEE Trans. Microw. Theory Tech. 63(3), 1097-1107 (2015)

4. R Nitzberg, Effect of a few dominant specular reflectors target model upon target detection. IEEE Trans. Aerosp. Electron. Syst. AES-14(4), 670-673 (1978)

5. K Gerlach, MJ Steiner, FC Lin, Detection of a spatially distributed target in white noise. IEEE Signal Process. Lett. 4(7), 198-200 (1997)

6. K Gerlach, MJ Steiner, Adaptive detection of range distributed targets. IEEE Trans. Signal Process. 47(7), 1844-1851 (1999)

7. T Jian, Y He, F Su, C Qu, Cascaded detector for range-spread target in non-Gaussian clutter. IEEE Trans. Aerosp. Electron. Syst. 48(2), $1713-1725(2012)$

8. S Xu, P Shui, X Yan, CFAR detection of range-spread target in white Gaussian noise using wave form entropy. Electron. Lett. 46(9), 647-649 (2010)

9. A Aubry, A De Maio, L Pallotta, A Farina, Radar detection of distributed targets in homogeneous interference whose inverse covariance structure is defined via unitary invariant functions. IEEE Trans. Signal Process. 61(20), 4949-4961 (2013)

10. T Jian, Y He, F Su, C Qu, Adaptive range-spread target detection based on modified generalized likelihood ratio test in non-Gaussian clutter. IET Radar Sonar Navig. 5(9), 970-977 (2011)

11. E Conte, AD Maio, G Ricci, GLRT-based adaptive detection algorithms for range-spread targets. IEEE Trans. Signal Process. 49(7), 1336-1348 (2001)

12. $\mathrm{Y} \mathrm{He}, \mathrm{T}$ Jian, $\mathrm{F} \mathrm{Su}, \mathrm{X} \mathrm{Gu}$, Novel range-spread target detectors in non-Gaussian clutter. IEEE Trans. Aerosp. Electron. Syst. 46(3), 1312-1328 (2010)

13. JL Sanz-González, F Álvarez-Vaquero, Nonparametric rank detectors under K-distributed clutter in radar applications. IEEE Trans. Aerosp. Electron. Syst. 41(2), 702-710 (2005)

14. H Chen, PK Varshney, S Kay, JH Michels, Noise enhanced nonparametric detection. IEEE Trans. Inf. Theory. 55(2), 499-506 (2009)

15. W Sun, S He, G Guo, W Li, Z He, Adaptive range-cell integrated detection method with application. Acta Electronica Sinica. 27(2), 111-112 (1999)

16. N Otsu, A threshold selection method from gray-level histograms. IEEE Trans. Syst. Man Cybernet. 9(1), 62-66 (1979)

17. EK Al-Hussaini, AM Al-Bassiouni, YA El-Far, Decentralized CFAR signal detection. Signal Process. 44(1995), 299-307 (1995)

18. B Zhang, Y Pi, J Li, Terahertz imaging radar with inverse aperture synthesis techniques: system structure, signal processing, and experiment results. IEEE Sensors J. 15(1), 290-299 (2015)

\section{Submit your manuscript to a SpringerOpen ${ }^{\circ}$ journal and benefit from:}

- Convenient online submission

- Rigorous peer review

- Immediate publication on acceptance

- Open access: articles freely available online

- High visibility within the field

- Retaining the copyright to your article

Submit your next manuscript at $\gg$ springeropen.com 